\title{
A convergence analysis of the numerical solution of boundary-value problems by using two-dimensional Haar wavelets
}

\author{
Narongpol Wichailukkana ${ }^{\mathrm{a}, *}$, Boriboon Novaprateep ${ }^{\mathrm{a}, \mathrm{b}}$, Chaiwoot Boonyasiriwat ${ }^{\mathrm{c}}$ \\ a Department of Mathematics, Faculty of Science, Mahidol University, Rama 6 Road, Ratchathewi, \\ Bangkok 10400 Thailand \\ b PERDO, Centre of Excellence in Mathematics, Commission on Higher Education, Faculty of Science, \\ Mahidol University, Rama 6 Road, Ratchathewi, Bangkok 10400 Thailand \\ c Department of Physics, Faculty of Science, Mahidol University, Rama 6 Road, Ratchathewi, \\ Bangkok 10400 Thailand
}

*Corresponding author, e-mail: narongpol.wic@student.mahidol.ac.th

Received 17 Mar 2016

Accepted 29 Sep 2016

\begin{abstract}
This study provides an analysis of the convergence of the Haar wavelet-based method for solving twodimensional boundary value problems. The convergence analysis shows that the approximation method is of order 2 . The analytical results are validated via two numerical examples.
\end{abstract}

KEYWORDS: Riemann-Liouville integral, partial differential equations, orthogonality, error of approximation

MSC2010: 65N12

\section{INTRODUCTION}

Partial differential equations (PDEs) can be solved numerically using various methods such as finiteelement, finite-difference, and finite volume methods. Wavelet-based methods have also been introduced for solving PDEs. These include a simple and effective wavelet-based method for solving differential equations in which the highest derivative is approximated by a wavelet series ${ }^{1}$.

Chen and Hsiao ${ }^{1,2}$ presented an operational matrix of integration based on Haar wavelets and a procedure for applying the matrix in order to analyse lumped and distributed-parameter dynamical systems. They recommended expanding the highest derivative appearing in the differential equation into the Haar wavelet series. The other derivatives and the solution function are then calculated through integration. All derivatives and the solution function are substituted into the ODE system. The ODE system is then discretized by the collocation method to form a linear system of algebraic equations in order to calculate the wavelet coefficients. By increasing the multiresolution parameter $m$, the accuracy of solution can be improved. Lepik adapted the method of Chen and Hsiao ${ }^{1}$ to solve various types of differential equations such as nonlinear ODEs ${ }^{3}$, evo- lution equations ${ }^{4}$, integral equations ${ }^{5}$, higher-order $\mathrm{ODEs}^{6}$, and PDEs ${ }^{3,7}$. Lepik ${ }^{7}$ proposed a procedure to solve PDEs by using the two-dimensional Haar wavelet and claimed that the proposed method was mathematically simple and computationally efficient for solving the diffusion and Poisson equations. The main feature is to expand the highest derivative into the 2-dimensional Haar wavelet series.

Although many wavelet methods have been proposed, little on convergence analysis has been published. Convergence analysis is important for determining the efficiency of a numerical method. Siraj-ul-Islam et $\mathrm{al}^{8,9}$ proved the convergence of the Haar wavelet series. Majak et al ${ }^{10,11}$ published a convergence theorem for solving ODEs using the wavelet method ${ }^{1,2}$. To the best of our knowledge, the convergence analysis of a wavelet-based method for solving PDEs has not been presented before. Since the method of Lepik ${ }^{7}$ is simple and efficient, we perform a convergence analysis on this method when applied to solve 2D PDE boundary value problems.

The convergence theorem presented here only holds for PDEs with boundary value problems. The validity of the convergence theorem is verified by two numerical examples in which the Poisson and Helmholtz equations are solved. These two ex- 
amples are used in this study because numerous scientific problems can be described by these two equations.

\section{THE HAAR WAVELET AND ITS PROPERTIES}

In this work, the Haar wavelet family is defined in the same way as in Ref. 1.

Definition 1 Consider the interval $[A, B]$, where $A$ and $B$ are given constants. Let $M=2^{J}$ and $m=2^{j}$ where $J$ is the maximum level of resolution and $j=$ $0,1, \ldots, J$ is a dilation parameter. $k=0,1, \ldots, m-1$ is the translation parameter. When $i>1$, the $i$ th Haar wavelet function is defined as

$$
h_{i}(x)= \begin{cases}1, & x \in\left[\xi_{1}(i), \xi_{2}(i)\right] \\ -1, & x \in\left[\xi_{2}(i), \xi_{3}(i)\right] \\ 0, & \text { elsewhere }\end{cases}
$$

where $i=2^{j}+k+1, \xi_{1}(i)=A+2 k \mu \Delta x, \xi_{2}(i)=$ $A+(2 k+1) \mu \Delta x, \xi_{3}(i)=A+2(k+1) \mu \Delta x, \mu=M / m$, and $\Delta x=(B-A) / 2 M$. The index $i$ is the wavelet number. The case $i=1$ corresponds to the scaling function of the Haar wavelet which is defined as

$$
h_{1}(x)=1, \quad x \in[A, B] .
$$

In this definition, $h_{2}(x)$ is called the Haar mother wavelet.

The Riemann-Liouville integral,

$$
\begin{aligned}
p_{\alpha, i}(x) & =\int_{A}^{x} \cdots \int_{A}^{x} h_{i}(t) \mathrm{d}^{\alpha} t \\
& =\frac{1}{(\alpha-1) !} \int_{A}^{x}(x-t)^{\alpha-1} h_{i}(t) \mathrm{d} t
\end{aligned}
$$

for $\alpha, i \in \mathbb{N}$, is required in order to solve $n$ th-order PDEs. The details of this integral is given in Ref. 12. When $\alpha=0$, we set

$$
p_{0, i}(x)=h_{i}(x) \text {. }
$$

Using (2) and (1) in (3) yields

$$
\begin{aligned}
& p_{\alpha, 1}(x)=\frac{1}{\alpha !}(x-A)^{\alpha}, \\
& p_{\alpha, i}(x)= \begin{cases}0, & x<\xi_{1}(i), \\
\frac{1}{\alpha !}\left[x-\xi_{1}(i)\right]^{\alpha}, & x \in\left[\xi_{1}(i), \xi_{2}(i)\right], \\
\frac{1}{\alpha !}\left\{\left[x-\xi_{1}(i)\right]^{\alpha},\right. & \\
\left.-2\left(x-\xi_{2}(i)\right)^{\alpha}\right\}, & x \in\left[\xi_{2}(i), \xi_{3}(i)\right], \\
\frac{1}{\alpha !}\left\{\left[x-\xi_{1}(i)\right]^{\alpha},\right. & \\
-2\left[x-\xi_{2}(i)\right]^{\alpha} & \\
\left.+\left[x-\xi_{3}(i)\right]^{\alpha}\right\}, & x>\xi_{3}(i),\end{cases}
\end{aligned}
$$

respectively. The Haar wavelets are orthogonal:

$$
\int_{A}^{B} h_{i}(x) h_{i^{\prime}}(x) \mathrm{d} x= \begin{cases}B-A, & i=i^{\prime}=1, \\ (B-A) / 2^{j}, & i=i^{\prime}>1, \\ 0, & i \neq i^{\prime},\end{cases}
$$

where $i=2^{j}+k+1, i^{\prime}=2^{j^{\prime}}+k^{\prime}+1$, and $i^{\prime}, j^{\prime}, k^{\prime}$ are defined in the same way as $i, j, k$, respectively. Since a Haar wavelet function is in $L^{2}[A, B]$, any function $f(x) \in L^{2}[A, B]$ can be expanded into a Haar wavelet series via

$$
f(x)=\sum_{i=1}^{\infty} a_{i} h_{i}(x) ; a_{i}=2^{j} \int_{A}^{B} f(x) h_{i}(x) \mathrm{d} x .
$$

The expansion in (8) can be rewritten as

$$
f(x)=a_{1} h_{1}(x)+\sum_{j=0}^{\infty} \sum_{k=0}^{2^{j}-1} a_{2^{j}+k+1} h_{2^{j+k+1}}(x),
$$

and can be approximated at resolution $J$ as

$$
f_{J}(x)=a_{1} h_{1}(x)+\sum_{j=0}^{J} \sum_{k=0}^{2^{j}-1} a_{2^{j}+k+1} h_{2^{j}+k+1}(x) .
$$

By using (9) and (10), we can define the error of approximation as

$$
\begin{aligned}
E_{J}(x) & =\left|f(x)-f_{J}(x)\right| \\
& =\left|\sum_{j=J+1}^{\infty} \sum_{k=0}^{2^{j}-1} a_{2^{j}+k+1} h_{2^{j}+k+1}(x)\right| .
\end{aligned}
$$

\section{NUMERICAL SOLUTION OF A PDE USING 2D HAAR WAVELETS}

This section briefly reviews the method proposed by Lepik ${ }^{7}$ for solving PDEs using 2D Haar wavelets. Consider the linear PDE,

$$
\begin{gathered}
\sum_{\gamma=0}^{\Gamma} \sum_{\lambda=0}^{\Lambda} D_{\gamma \lambda}(x, y) \frac{\partial^{\gamma+\lambda}}{\partial x^{\gamma} \partial y^{\lambda}} u(x, y)=f(x, y), \\
(x, y) \in \Omega, \quad \partial \Omega=\sigma,
\end{gathered}
$$

where $\Gamma, \Lambda$ are given constants, and $D_{\gamma \lambda}(x, y)$ and $f(x, y)$ are given functions. The quantities $\Gamma$ and $\Lambda$ can be determined, respectively, from the maximum order of the $x$ and $y$ derivatives appearing in the linear system (12). By simplifying the system, the domain $\Omega$ is considered as a rectangular domain $\left[A_{1}, B_{1}\right] \times\left[A_{2}, B_{2}\right]$. The intervals $\left[A_{1}, B_{1}\right]$ and $\left[A_{2}, B_{2}\right]$ are divided into $2 M_{1}\left(=2^{J+1}\right)$ and $2 M_{2}(=$ $2^{J^{\prime}+1}$ ) parts of equal length, respectively. 
If (12) holds for $\partial^{\Gamma+\Lambda} u(x, y) / \partial x^{\Gamma} \partial y^{\Lambda} \in L^{2}(\Omega)$, we can approximate that

$$
\begin{aligned}
\frac{\partial^{\Gamma+\Lambda} u(x, y)}{\partial x^{\Gamma} \partial y^{\Lambda}} & =\sum_{i=1}^{\infty} \sum_{i^{\prime}=1}^{\infty} a_{i i^{\prime}} h_{i}(x) h_{i^{\prime}}(y) \\
& \approx \sum_{i=1}^{2 M_{1}} \sum_{i^{\prime}=1}^{2 M_{2}} a_{i i^{\prime}} h_{i}(x) h_{i^{\prime}}(y),
\end{aligned}
$$

where $a_{i i^{\prime}}$ are wavelet coefficients, and $h_{i}(x), h_{i^{\prime}}(y)$ are Haar functions. Then the solution $u(x, y)$ can be obtained by taking the integrals in (3) $\Gamma$ and $\Lambda$ times with respect to $x$ and $y$, respectively. In this process, the unknown functions can be obtained using the boundary conditions $\sigma$. The solution $u(x, y)$ will appear in the form:

$$
u(x, y)=\sum_{i=1}^{\infty} \sum_{i^{\prime}=1}^{\infty} a_{i i^{\prime}} p_{\Gamma, i}(x) p_{\Lambda, i^{\prime}}(y)+\Psi(x, y),
$$

which can be approximated by

$$
u_{J J^{\prime}}(x, y)=\sum_{i=1}^{2 M_{1}} \sum_{i^{\prime}=1}^{2 M_{2}} a_{i i^{\prime}} p_{\Gamma, i}(x) p_{\Lambda, i^{\prime}}(y)+\Psi(x, y),
$$

where $p_{\Gamma, i}(x), p_{\Lambda, i^{\prime}}(y)$ are functions defined in (5) and (6), and $\Psi(x, y)$ is a function satisfying the boundary conditions $\sigma$. The other derivatives can be directly determined by taking the derivatives of $u(x, y)$.

The collocation points $\left(x_{r}, y_{s}\right)$ are defined by

$$
\left(x_{r}, y_{s}\right)=\left(\frac{(2 r-1) \Delta_{1}}{4 M_{1}}, \frac{(2 s-1) \Delta_{2}}{4 M_{2}}\right),
$$

where $\Delta_{1}=B_{1}-A_{1} ; \Delta_{2}=B_{2}-A_{2} ; r=1, \ldots, 2 M_{1}$ and $s=1, \ldots, 2 M_{2}$. By substituting the approximate solution (15) and its derivatives at the collocation points (16) into (12), we obtain the system of linear equations

$$
\sum_{i=0}^{2 M_{1}} \sum_{i^{\prime}=0}^{2 M_{2}} a_{i i^{\prime}} R_{i i^{\prime} r s}=f\left(x_{r}, y_{s}\right) .
$$

The wavelet coefficients $a_{i i^{\prime}}$ can be calculated from (17). However, dealing with a fourth-order matrix equation is complicated. For convenience, we transform the fourth-order matrix equation to a secondorder matrix equation. After the $a_{i i^{\prime}}$ are calculated, we substitute them back into (15) to obtain the solution ${ }^{7}$.

\section{CONVERGENCE ANALYSIS}

We derive the upper bound of $p_{\alpha, i}(x)$ since the upper bound is needed to derive the convergence theorem.

Theorem 1 Suppose $p_{\alpha, i}(x)$ is defined as in (5) and (6) on $[0,1]$. Then the upper bound of $p_{\alpha, i}(x)$ is as follows:

$$
\begin{aligned}
& p_{0, i}(x) \leqslant 1, \quad p_{\alpha, 1}(x) \leqslant \frac{1}{\alpha !}, \\
& p_{1, i}(x) \leqslant \frac{1}{2^{j+1}} \quad \text { when } i>1, \\
& p_{\alpha, i}(x)<C(\alpha)\left(\frac{1}{2^{j+1}}\right)^{2} \text { when } \alpha \leqslant 2, i>1,
\end{aligned}
$$

where $C(\alpha)=8 /\left(3(\lfloor(\alpha+1) / 2\rfloor !)^{2}\right)$.

Proof: Suppose that $p_{\alpha, i}(x)$ is defined as in (5) and $(6)$ on $[0,1]$. According to Definition 1 , we have $\xi_{1}(i)=2 k / 2^{j+1}, \xi_{2}(i)=(2 k+1) / 2^{j+1}$ and $\xi_{3}(i)=2(k+1) / 2^{j+1}$, since $p_{\alpha, i}(x)$ is defined on $[0,1]$. Then, we have

$$
\xi_{2}(i)-\xi_{1}(i)=\xi_{3}(i)-\xi_{2}(i)=\frac{1}{2^{j+1}} .
$$

According to (4), $p_{0, i}(x)$ is a Haar wavelet defined in Definition 1 so it is obvious that

$$
-1 \leqslant p_{0, i}=h_{i}(x) \leqslant 1, \quad \forall i .
$$

We now find the upper bound for $p_{1, i}(x)$. By taking $\mathrm{d} / \mathrm{d} x$ on $p_{1,1}(x)$ defined as $(5)$ on $[0,1]$, we obtain

$$
\frac{\mathrm{d} p_{1,1}(x)}{\mathrm{d} x}=1,
$$

so $p_{1,1}(x)$ is non-decreasing on $[0,1]$. Then $p_{1,1}(x) \leqslant 1$. We then find the upper bound for $p_{1, i}$ when $i>1$. Taking $\mathrm{d} / \mathrm{d} x$ on $p_{1, i}(x)$ defined as (6) on $[0,1]$ yields

$$
\begin{array}{ll}
\frac{\mathrm{d}}{\mathrm{d} x} p_{1, i}(x)=0, & x \in\left[0, \xi_{1}(i)\right], \\
\frac{\mathrm{d}}{\mathrm{d} x} p_{1, i}(x)=1>0, & x \in\left[\xi_{1}(i), \xi_{2}(i)\right], \\
\frac{\mathrm{d}}{\mathrm{d} x} p_{1, i}(x)=-1<0, & x \in\left[\xi_{2}(i), \xi_{3}(i)\right], \\
\frac{\mathrm{d}}{\mathrm{d} x} p_{1, i}(x)=0, & x \in\left[\xi_{3}(i), 1\right] .
\end{array}
$$

Equations (19)-(22) imply that $p_{1, i}(x)$ is nondecreasing when $x \in\left[0, \xi_{2}(i)\right]$ and then nonincreasing when $x \in\left[\xi_{2}(i), 1\right]$. By (6), $p_{1, i}(x)$ is 
continuous, so $p_{1, i}(x)$ is a maximum at $x=\xi_{2}(i)$. Then

$$
\begin{aligned}
p_{1, i}(x) \leqslant \xi_{2}(i)-\xi_{1}(i)=\frac{1}{2^{j+1}}, & \forall x \in[0,1], \\
& \forall i>1 . \quad
\end{aligned}
$$

By taking $\mathrm{d} / \mathrm{d} x$ on $p_{\alpha, 1}(x)$, we obtain

$$
\frac{\mathrm{d}}{\mathrm{d} x} p_{\alpha, 1}(x)=\frac{1}{(\alpha-1) !} x^{\alpha-1} \geqslant 0,
$$

so $p_{\alpha, 1}(x)$ is non-decreasing over $x \in[0,1]$ and hence a maximum at $x=1$. Hence

$$
p_{\alpha, 1}(x) \leqslant \frac{1}{\alpha !} \text { when } \quad \alpha \geqslant 2 .
$$

Taking $\mathrm{d} / \mathrm{d} x$ on $p_{\alpha, i}(x)$ when $\alpha \geqslant 2$ and $i>1$ yields

$$
\begin{array}{rr}
\frac{\mathrm{d}}{\mathrm{d} x} p_{\alpha, i}(x)=0, & x \in\left[0, \xi_{1}(i)\right], \\
\frac{\mathrm{d}}{\mathrm{d} x} p_{\alpha, i}(x)=\frac{1}{(\alpha-1) !}\left[x-\xi_{1}(i)\right]^{\alpha-1} \geqslant 0, \\
x \in\left[\xi_{1}(i), \xi_{2}(i)\right], \\
\frac{\mathrm{d}}{\mathrm{d} x} p_{\alpha, i}(x)=\frac{1}{(\alpha-1) !}\left\{\left[x-\xi_{1}(i)\right]^{\alpha-1}\right. \\
\left.-2\left[x-\xi_{2}(i)\right]^{\alpha-1}\right\}, \\
x \in\left[\xi_{2}(i), \xi_{3}(i)\right] .
\end{array}
$$

To show that $p_{\alpha, i}(x)$ is non-decreasing for every $x \in$ $[0,1]$ when $\alpha \geqslant 2$, we suppose that $(\mathrm{d} / \mathrm{d} x) p_{\alpha, i} \geqslant 0$ when $\alpha \geqslant 2, i>1$ and $x \in\left[\xi_{2}(i), \xi_{3}(i)\right]$, so we have

$$
\left[x-\xi_{1}(i)\right]^{\alpha-1} \geqslant 2\left[x-\xi_{2}(i)\right]^{\alpha-1} .
$$

By rearranging (27) and applying (18), we obtain

$$
x \leqslant \xi_{2}(i)+\left[\xi_{3}(i)-\xi_{2}(i)\right]\left(\frac{1}{2^{1 /(\alpha-1)}-1}\right) .
$$

Then $p_{\alpha, i}(x)$ defined in (6) when $x \in$ $\left[\xi_{2}(i), \xi_{3}(i)\right]$ is non-decreasing when $x \leqslant \xi_{2}(i)+\left[\xi_{3}(i)-\xi_{2}(i)\right]\left(1 /\left(2^{1 /(\alpha-1)}-1\right)\right)$. Since $\xi_{3}(i)=\xi_{2}(i)+\left[\xi_{3}(i)-\xi_{2}(i)\right] \leqslant$ $\xi_{2}(i)+\left[\xi_{3}(i)-\xi_{2}(i)\right]\left(1 /\left(2^{1 /(\alpha-1)}-1\right)\right), p_{\alpha, i}(x)$ is non-decreasing when $x \in\left[\xi_{2}(i), \xi_{3}(i)\right]$ and $\alpha \geqslant 2$. In the subinterval $x \in\left[\xi_{3}(i), 1\right]$, by using the binomial expansion, $p_{\alpha, i}$ can be rewritten as

$$
\begin{aligned}
& p_{\alpha, i}(x)= \\
& \frac{1}{\alpha !}\left\{\sum_{l=0}^{\alpha}\left(\begin{array}{l}
\alpha \\
l
\end{array}\right)\left[x-\xi_{2}(i)\right]^{\alpha-l}\left[\xi_{2}(i)-\xi_{1}(i)\right]^{l}\right. \\
& -2\left[x-\xi_{2}(i)\right]^{\alpha} \\
& \left.+\sum_{l=0}^{\alpha}\left(\begin{array}{l}
\alpha \\
l
\end{array}\right)\left[x-\xi_{2}(i)\right]^{\alpha-l}\left[\xi_{2}(i)-\xi_{3}(i)\right]^{l}\right\} \text {. }
\end{aligned}
$$

Substituting (18) into (29) and rearranging yields

$$
\begin{aligned}
p_{\alpha, i}(x)=\frac{1}{\alpha !} \sum_{l=2}^{\alpha} & \left\{\left(\begin{array}{l}
\alpha \\
l
\end{array}\right)\left[x-\xi_{2}(i)\right]^{\alpha-l}\right. \\
\cdot & {\left.\left[\left(\frac{1}{2^{j+1}}\right)^{l}+\left(-\frac{1}{2^{j+1}}\right)^{l}\right]\right\}, }
\end{aligned}
$$

where $\alpha \geqslant 2$ and $i>1$. The term $\left[\left(1 /\left(2^{j+1}\right)\right)^{l}+\right.$ $\left.\left(-1 /\left(2^{j+1}\right)\right)^{l}\right]$ from (30) equals zero when $l$ is odd and is larger than zero when $l$ is even. Thus

$$
\begin{aligned}
\frac{\mathrm{d}}{\mathrm{d} x} p_{\alpha, i}(x) & =\frac{2}{\alpha !} \sum_{l=2}^{\alpha}\left(\begin{array}{l}
\alpha \\
l
\end{array}\right) \frac{(\alpha-l)\left[x-\xi_{2}(i)\right]^{\alpha-l-1}}{\left(2^{j+1}\right)^{l}} \\
& \geqslant 0,
\end{aligned}
$$

when $\alpha, i>1$ and $l$ is even. Hence, $p_{\alpha, i}(x)$ is nondecreasing when $x \in\left[\xi_{3}(i), 1\right], \alpha \geqslant 2$ and $i>1$. Thus $p_{\alpha, i}(x)$ is non-decreasing for $x \in[0,1]$ when $\alpha \geqslant 2$ and $i>1$, and has a maximum at $x=1$.

Finally, we find the upper bound of $p_{\alpha, i}(x)$ defined by (6) at $x=1$ when $\alpha \geqslant 2$ and $i>1$. It is obvious that

$$
\left(\begin{array}{l}
\alpha \\
l
\end{array}\right) \leqslant\left(\begin{array}{l}
\alpha \\
\bar{\alpha}
\end{array}\right)=\frac{\alpha !}{\bar{\alpha} !(\alpha-\bar{\alpha}) !}=\frac{\alpha !}{(\bar{\alpha} !)^{2}},
$$

where $\alpha \geqslant l$ and $\bar{\alpha}=\lfloor(\alpha+1) / 2\rfloor$. By investigating the width of the subinterval $\left[\xi_{2}(i), 1\right]$, we have

$$
\left[x-\xi_{2}(i)\right]^{\alpha-l} \leqslant\left[1-\xi_{2}(i)\right]^{\alpha-l} \leqslant 1,
$$

where $\alpha-l \geqslant 0$ and $x \in\left[\xi_{3}(i), 1\right]$. By considering (32) and (33), $p_{\alpha, i}(x)$ in (30) is bounded by the following:

$$
p_{\alpha, i}(x) \leqslant \frac{2}{(\bar{\alpha} !)^{2}} \sum_{l=2}^{\alpha}\left(\frac{1}{2^{j}}\right)^{l}\left(\frac{1}{2}\right)^{l},
$$

where $l$ is even. Since $\left(1 / 2^{j}\right)^{2}>\left(1 / 2^{j}\right)^{3}>\ldots>$ $\left(1 / 2^{j}\right)^{l}>\ldots$, (34) becomes

$$
\begin{aligned}
p_{\alpha, i}(x) & <\frac{8}{(\bar{\alpha} !)^{2}}\left(\frac{1}{2^{j+1}}\right)^{2}\left[\sum_{\rho=0}^{\lfloor\alpha / 2\rfloor}\left(\frac{1}{2}\right)^{2 \rho}-1\right] \\
& <\frac{8}{(\bar{\alpha} !)^{2}}\left(\frac{1}{2^{j+1}}\right)^{2}\left[\sum_{\rho=0}^{\infty}\left(\frac{1}{2}\right)^{2 \rho}-1\right] \\
& =\frac{8}{3(\bar{\alpha} !)^{2}}\left(\frac{1}{2^{j+1}}\right)^{2} .
\end{aligned}
$$

Hence

$$
p_{\alpha, i}(x)<C(\alpha)\left(\frac{1}{2^{j+1}}\right)^{2}, \quad \forall x \in[0,1],
$$

where $C(\alpha)=8 /\left(3(\lfloor(\alpha+1) / 2\rfloor !)^{2}\right), \alpha \geqslant 2$ and $i>$ 1. 
Definition 2 According to Lepik ${ }^{7}$, the solution of a 2D PDE of a boundary value problem is

$$
u(x, y)=\sum_{i=1}^{\infty} \sum_{i^{\prime}=1}^{\infty} a_{i i^{\prime}} p_{\Gamma, i}(x) p_{\Lambda, i^{\prime}}(y)+\Psi(x, y),
$$

and (36) can be approximated with the maximum level of resolution $J$ and $J^{\prime}$ as

$$
u_{J J^{\prime}}(x, y)=\sum_{i=1}^{2 M_{1}} \sum_{i^{\prime}=1}^{2 M_{2}} a_{i i^{\prime}} p_{\Gamma, i}(x) p_{\Lambda, i^{\prime}}(y)+\Psi(x, y),
$$

where $M_{1}=2^{J}$ and $M_{2}=2^{J^{\prime}}$. Then, the error of the approximation at the maximal level of resolution $J$ and $J^{\prime}$ is defined as

$$
E_{J J^{\prime}}=\left|u(x, y)-u_{J J^{\prime}}(x, y)\right| .
$$

Definition 3 By Definition 2, the $L^{2}$-norm of the error of the approximation at the maximum level of resolution $J$ and $J^{\prime}$ can be defined as

$$
\left\|E_{J J^{\prime}}(x, y)\right\|_{2}=\left\{\iint_{\mathbb{D}}\left[E_{J J^{\prime}}(x, y)\right]^{2} \mathrm{~d} x \mathrm{~d} y\right\}^{1 / 2},
$$

where $(x, y) \in \mathbb{D}$ and $\mathbb{D}$ is a rectangular domain. Then $\left\|E_{J J^{\prime}}(x, y)\right\|_{2}$ is called the $L^{2}$-norm of the error.

Theorem 2 Given $\Gamma, \Lambda \geqslant 2$, assume that

$$
K(x, y)=\frac{\partial^{(\Gamma+\Lambda)}}{\partial x^{\Gamma} \partial y^{\Lambda}} u(x, y) \in L^{2}\left(\mathbb{R}^{2}\right)
$$

is a continuous function on $[0,1]^{2}$ and can be approximated as

$$
K(x, y) \approx \sum_{i=1}^{2 M_{1}} \sum_{i^{\prime}=1}^{2 M_{2}} a_{i i^{\prime}} h_{i}(x) h_{i^{\prime}}(y),
$$

where $M_{1}=2^{J}$ and $M_{2}=2^{J^{\prime}}$. For all $(x, y) \in[0,1]^{2}$, there exists $\omega \geqslant 0$ such that

$$
|K|,\left|\frac{\partial}{\partial x} K\right|,\left|\frac{\partial}{\partial y} K\right|,\left|\frac{\partial^{2}}{\partial x \partial y} K\right| \leqslant \omega ;
$$

where $K=K(x, y)$. Then the Haar wavelet method, based on Ref. 7 , is convergent, and it yields the following properties for a boundary value problem on domain $[0,1]^{2}$ of a $2 D$ PDE. Let $\left\|E_{J J^{\prime}}(x, y)\right\|_{2}$ be the $L^{2}$-norm of the error at the maximum level of resolution $J$ and $J^{\prime}$ given in Definition 3. Then

$$
\left\|E_{J J^{\prime}}(x, y)\right\|_{2}<G(\Gamma, \Lambda)\left(\frac{1}{2^{\tilde{J}+1}}\right)^{2},
$$

or

$$
\left\|E_{J J^{\prime}}(x, y)\right\|_{2}=O\left(\left(\frac{1}{2^{\tilde{J}+1}}\right)^{2}\right) ; \quad \Gamma, \Lambda \geqslant 2,
$$

where

$$
\begin{aligned}
G(\Gamma, \Lambda)=\{ & \frac{[C(\Lambda)]^{2}}{36(\Gamma !)^{2}}+\frac{[C(\Gamma)]^{2}}{36(\Lambda !)^{2}} \\
& +\frac{[C(\Gamma)]^{2}[C(\Lambda)]^{2}}{1296}+\frac{C(\Gamma) C(\Lambda)}{8 \Gamma ! \Lambda !} \\
& \left.+\frac{[C(\Gamma)]^{2} C(\Lambda)}{108 \Lambda !}+\frac{C(\Gamma)[C(\Lambda)]^{2}}{108 \Gamma !}\right\}^{1 / 2} \omega,
\end{aligned}
$$

$C(\Gamma)=8 /\left[3(\lfloor(\Gamma+1) / 2\rfloor)^{2}\right]$ for $n \geqslant 2$ and $\tilde{J}=$ $\min \left\{J, J^{\prime}\right\}$.

Proof: According to Lepik ${ }^{7}$, the solution of a 2D PDE is in the form

$$
u(x, y)=\sum_{i=1}^{\infty} \sum_{i^{\prime}=1}^{\infty} a_{i i^{\prime}} p_{\Gamma, i}(x) p_{\Lambda, i^{\prime}}(y)+\Psi(x, y),
$$

where $\Psi(x, y)$ is a function determined by the boundary conditions, and (39) can be rewritten as

$$
\begin{array}{r}
u(x, y)=a_{11} p_{\Gamma, 1}(x) p_{\Lambda, 1}(y)+\Psi(x, y) \\
+\sum_{j=0}^{\infty} \sum_{k=0}^{2^{j}-1} a_{2^{j}+k+1,1} p_{\Gamma, 2^{j}+k+1}(x) p_{\Lambda, 1}(y) \\
+\sum_{j^{\prime}=0}^{\infty} \sum_{k^{\prime}=0}^{j^{j^{\prime}}-1} a_{1,2^{j^{\prime}+k^{\prime}+1}} p_{\Gamma, 1}(x) p_{\Lambda, 2^{j^{\prime}}+k^{\prime}+1}(y) \\
+\sum_{j=0}^{\infty} \sum_{k=0}^{2^{j}-1} \sum_{j^{\prime}=0}^{\infty} \sum_{k^{\prime}=0}^{2^{j^{\prime}}-1} a_{2^{j}+k+1,2^{j^{\prime}}+k^{\prime}+1} \\
\cdot p_{\Gamma, 2^{j}+k+1}(x) p_{\Lambda, 2^{j^{\prime}}+k^{\prime}+1}(y),
\end{array}
$$

where $i=2^{j}+k+1$ and $i^{\prime}=2^{j^{\prime}}+k^{\prime}+1$. Then, the approximate solution at the maximum level of resolution $J$ and $J^{\prime}$ relevant to $p_{\Gamma, i}(x)$ and $p_{\Lambda, i^{\prime}}(y)$, respectively, is

$$
\begin{array}{r}
u_{J J^{\prime}}(x, y)=a_{11} p_{\Gamma, 1}(x) p_{\Lambda, 1}(y)+\Psi(x, y) \\
+\sum_{j=0}^{J} \sum_{k=0}^{2^{j}-1} a_{2^{j}+k+1,1} p_{\Gamma, 2^{j}+k+1}(x) p_{\Lambda, 1}(y) \\
+\sum_{j^{\prime}=0}^{J^{\prime}} \sum_{k^{\prime}=0}^{2^{j^{\prime}}-1} a_{1,2^{j^{\prime}+k^{\prime}+1}} p_{\Gamma, 1}(x) p_{\Lambda, 2^{j^{\prime}}+k^{\prime}+1}(y) \\
+\sum_{j=0}^{J} \sum_{k=0}^{2^{j}-1} \sum_{j^{\prime}=0}^{J^{\prime}} \sum_{k^{\prime}=0}^{2^{j^{\prime}}-1} a_{2^{j}+k+1,2^{j^{\prime}}+k^{\prime}+1} \\
\cdot p_{\Gamma, 2^{j}+k+1}(x) p_{\Lambda, 2^{j^{\prime}}+k^{\prime}+1}(y),
\end{array}
$$


where $i=2^{j}+k+1$ and $i^{\prime}=2^{j^{\prime}}+k^{\prime}+1$. By Definition 2, the error of approximation $E_{J J^{\prime}}(x, y)=$ $\left|u(x, y)-u_{J J^{\prime}}(x, y)\right|$ with respect to (40) and (37) can be written as

$$
E_{J J^{\prime}}(x, y)=\left|T_{1}+T_{2}+T_{T_{3}}\right|,
$$

where

$$
\begin{aligned}
T_{1}= & \sum_{j=J+1}^{\infty} \sum_{k=0}^{2^{j}-1} a_{2^{j}+k+1,1} p_{\Gamma, 2^{j}+k+1}(x) p_{\Lambda, 1}(y) \\
T_{2}= & \sum_{j^{\prime}=J^{\prime}+1}^{\infty} \sum_{k^{\prime}=0}^{j^{j^{\prime}}-1} a_{1,2^{j^{\prime}+k^{\prime}+1}} p_{\Gamma, 1}(x) p_{\Lambda, 2^{j^{\prime}+k^{\prime}+1}}(y) \\
T_{3}= & \sum_{j=J+1}^{\infty} \sum_{k=0}^{2^{j}-1} \sum_{j^{\prime}=J^{\prime}+1}^{\infty} \sum_{k^{\prime}=0}^{2^{j^{\prime}}-1} a_{2^{j}+k+1,2^{j^{\prime}+k^{\prime}+1}} \\
& \cdot p_{\Gamma, 2^{j}+k+1}(x) p_{\Lambda, 2^{\prime}+k^{\prime}+1}(y),
\end{aligned}
$$

where $i=2^{j}+k+1$ and $i^{\prime}=2^{j^{\prime}}+k^{\prime}+1$. From Definition 3, the $L^{2}$-norm of the error $\left\|E_{J J^{\prime}}(x, y)\right\|_{2}$ can be written as

$$
\left\|E_{J J^{\prime}}(x, y)\right\|_{2}^{2}=\mathscr{D}_{1}+\mathscr{D}_{2}+\mathscr{D}_{3}+\mathscr{D}_{4}+\mathscr{D}_{5}+\mathscr{D}_{6}
$$

where

$$
\begin{aligned}
& \mathscr{D}_{1}=\sum_{j, k} \sum_{r, s} a_{2^{j}+k+1,1} a_{2^{r}+s+1,1} \mathscr{I}_{\Gamma ; j, k ; r, s}^{(2)} \mathscr{I}_{\Lambda}^{(1)}, \quad \\
& \mathscr{D}_{2}=\sum_{j^{\prime}, k^{\prime}} \sum_{r^{\prime}, s^{\prime}} a_{1, j^{j^{\prime}}+k^{\prime}+1} a_{1,2^{r^{\prime}+s^{\prime}+1}} \mathscr{I}_{\Gamma}^{(1)} \mathscr{I}_{\Lambda ; j^{\prime}, k^{\prime} ; r^{\prime}, s^{\prime}}^{(2)}
\end{aligned}
$$

$$
\begin{aligned}
\mathscr{D}_{3}= & \sum_{j, k} \sum_{r, s} \sum_{j^{\prime}, k^{\prime}} \sum_{r^{\prime}, s^{\prime}} a_{2^{j}+k+1,22^{\prime}+k^{\prime}+1} \\
& \cdot a_{2 r+s+1,2^{r^{\prime}+s^{\prime}+1}} \mathscr{I}_{\Gamma ; j, k ; r, s}^{(2)} \mathscr{I}_{\Lambda ; j^{\prime}, k^{\prime} ; r^{\prime}, s^{\prime}}^{(2)}, \\
\mathscr{D}_{4}=2 & \sum_{j, k} \sum_{r^{\prime}, s^{\prime}} a_{2 j^{j}+k+1,1} \\
& \cdot a_{1,2^{r^{\prime}+s^{\prime}+1}} \mathscr{I}_{\Gamma ; j, k}^{(3)} \mathscr{I}_{\Lambda ; r^{\prime}, s^{\prime}}^{(3)}, \\
\mathscr{D}_{5}=2 & \sum_{j, k} \sum_{r, s} \sum_{r^{\prime}, s^{\prime}} a_{2 j+k+1,1} a_{2 r^{r}+s+1, r^{\prime}+s^{\prime}+1} \\
& \cdot \mathscr{I}_{\Gamma ; j, k ; r, s^{2}}^{(2)} \mathscr{I}_{\Lambda ; r^{\prime}, s^{\prime}}^{(3)}, \\
\mathscr{D}_{6}=2 & \sum_{r, s} \sum_{j^{\prime}, k^{\prime}} \sum_{r^{\prime}, s^{\prime}} a_{1, j^{\prime}+k^{\prime}+1} a_{2^{r}+s+1,2^{\prime}+s^{\prime}+1} \\
& \cdot \mathscr{I}_{\Gamma ; r, s}^{(3)} \mathscr{I}_{\Lambda ; j^{\prime}, k^{\prime} ; r^{\prime}, s^{\prime}}^{(2)},
\end{aligned}
$$

where

$$
\begin{aligned}
\mathscr{I}_{\Gamma}^{(1)} & =\int_{0}^{1}\left[p_{\Gamma, 1}(x)\right]^{2} \mathrm{~d} x, \\
\mathscr{I}_{\Gamma ; j, k ; r, s}^{(2)} & =\int_{0}^{1}\left[p_{\Gamma, 2^{j}+k+1}(x) p_{\Gamma, 2^{r}+s+1}(x)\right] \mathrm{d} x, \\
\mathscr{I}_{\Gamma ; j, k}^{(3)} & =\int_{0}^{1}\left[p_{\Gamma, 2^{j}+k+1}(x) p_{\Gamma, 1}(x)\right] \mathrm{d} x,
\end{aligned}
$$

where $j=J+1, \ldots, \infty ; k=0,1, \ldots, 2^{j}-1 ; r=J+$ $1, \ldots, \infty ; s=0,1, \ldots, 2^{r}-1 ; j^{\prime}=J^{\prime}+1, \ldots, \infty ; k^{\prime}=$ $0,1, \ldots, 2^{j^{\prime}}-1 ; r^{\prime}=J^{\prime}+1, \ldots, \infty ; s^{\prime}=0,1, \ldots, 2^{r^{\prime}}-$ $1 ; j, j^{\prime}, r$, and $r^{\prime}$ are dilation parameters; and $k, k^{\prime}$, $s$, and $s^{\prime}$ are translation parameters.

The next step is to find the upper bound of $a_{i i^{\prime}}$ by considering

$$
K(x, y)=\sum_{i=1}^{2 M_{1}} \sum_{i^{\prime}=1}^{2 M_{2}} a_{i i^{\prime}} h_{i}(x) h_{i^{\prime}}(y)
$$

Since the problem of interest is a boundary value problem on domain $[0,1]^{2}$, both $p_{\Gamma, i}(x)$ and $p_{\Lambda, i}(y)$ are defined on $[0,1]$. Then, by Definition 1 , we have

$$
\xi_{2}(i)-\xi_{1}(i)=\xi_{3}(i)-\xi_{2}(i)=\frac{1}{2^{j+1}} .
$$

Since the domain is $[0,1]^{2},(7)$ becomes

$$
\int_{0}^{1} h_{i}(x) h_{i^{\prime}}(x) \mathrm{d} x= \begin{cases}1, & \text { when } i=i^{\prime}=1 \\ 2^{-j}, & \text { when } i=i^{\prime}>1 \\ 0, & \text { when } i \neq i^{\prime}\end{cases}
$$

Then we need to find the upper bound of $a_{11}$. By applying the orthogonal property (55) in (53) and setting $i, i^{\prime}=1$, we see that

$$
\begin{aligned}
a_{11} \int_{0}^{1} \int_{0}^{1} K(x, y) & h_{1}(x) h_{1}(y) \mathrm{d} x \mathrm{~d} y \\
& =\int_{0}^{1} \int_{0}^{1} K(x, y) \mathrm{d} x \mathrm{~d} y .
\end{aligned}
$$

Applying the mean value theorem for integrals with respect to $x$ and then $y$ to (56), we find

$$
a_{11}=\int_{0}^{1} K(\delta, y) \mathrm{d} y=K(\delta, \tilde{\delta}) \leqslant|K(\delta, \tilde{\delta})| \leqslant \omega,
$$

where $\delta, \tilde{\delta} \in[0,1]$. We then find the upper bound of $a_{i, 1}$ when $i>1$. By letting $\tilde{\varepsilon}_{3} \in(0,1), \varepsilon_{4} \in$ 
$\left(\xi_{1}(i), \xi_{2}(i)\right), \varepsilon_{5} \in\left(\xi_{2}(i), \xi_{3}(i)\right), \varepsilon_{6} \in\left(\varepsilon_{4}, \varepsilon_{5}\right)$ and $i=2^{j}+k+1$, where $\xi_{1}(i), \xi_{2}(i), \xi_{3}(i)$ are defined by Definition 1 , it yields

$$
\begin{aligned}
\left|\varepsilon_{4}-\varepsilon_{5}\right| & <\left|\varepsilon_{4}-\xi_{2}(i)\right|+\left|\xi_{2}(i)-\varepsilon_{5}\right| \\
& <\left|\xi_{1}(i)-\xi_{2}(i)\right|+\left|\xi_{2}(i)-\xi_{3}(i)\right| \\
& =\frac{1}{2^{j}},
\end{aligned}
$$

where $i=2^{j}+k+1$. Applying the orthogonal property (55) for $i>1$ and $i^{\prime}=1$ to (53) yields

$$
a_{i, 1}=2^{j} \int_{0}^{1} \int_{0}^{1} K(x, y) h_{i}(x) \mathrm{d} y \mathrm{~d} x .
$$

Applying the mean value theorem for integrals with respect to $y$ and then $x$ to (59), we obtain

$$
\begin{aligned}
a_{i, 1}=2^{j} & \int_{0}^{1} K\left(x, \tilde{\varepsilon}_{3}\right) h_{i}(x) \mathrm{d} x \\
=2^{j} & \left\{\int_{\xi_{1}(i)}^{\xi_{2}(i)} K\left(x, \tilde{\varepsilon}_{3}\right) \mathrm{d} x-\int_{\xi_{2}(i)}^{\xi_{3}(i)} K\left(x, \tilde{\varepsilon}_{3}\right) \mathrm{d} x\right\} \\
=2^{j} & \left\{\left[\xi_{2}(i)-\xi_{1}(i)\right] K\left(\varepsilon_{4}, \tilde{\varepsilon}_{3}\right)\right. \\
& \left.\quad-\left[\xi_{3}(i)-\xi_{2}(i)\right] K\left(\varepsilon_{5}, \tilde{\varepsilon}_{3}\right)\right\} .
\end{aligned}
$$

Substituting (54) into (60) yields

$$
a_{i, 1}=\frac{1}{2}\left[K\left(\varepsilon_{4}, \tilde{\varepsilon}_{3}\right)-K\left(\varepsilon_{5}, \tilde{\varepsilon}_{3}\right)\right] .
$$

Applying the mean value theorem to $K\left(\varepsilon_{4}, \tilde{\varepsilon}_{3}\right)$ and $K\left(\varepsilon_{5}, \tilde{\varepsilon}_{3}\right)$ in (61) yields

$$
a_{i, 1}=\frac{1}{2}\left(\varepsilon_{4}-\varepsilon_{5}\right) \frac{\partial}{\partial x} K\left(\varepsilon_{6}, \tilde{\varepsilon}_{3}\right) .
$$

(58) and (62) imply that

$$
a_{i, 1} \leqslant \frac{1}{2}\left|\varepsilon_{4}-\varepsilon_{5}\right|\left|\frac{\partial}{\partial x} K\left(\varepsilon_{6}, \tilde{\varepsilon}_{3}\right)\right|<\frac{\omega}{2^{j+1}} .
$$

By letting $\varepsilon_{3} \in(0,1), \quad \tilde{\varepsilon}_{4} \in\left(\xi_{1}\left(i^{\prime}\right), \xi_{2}\left(i^{\prime}\right)\right), \quad \tilde{\varepsilon}_{5} \in$ $\left(\xi_{2}\left(i^{\prime}\right), \xi_{3}\left(i^{\prime}\right)\right), \tilde{\varepsilon}_{6} \in\left(\tilde{\varepsilon}_{4}, \tilde{\varepsilon}_{5}\right), \varepsilon_{1} \in\left(\xi_{1}(i), \xi_{2}(i)\right), \varepsilon_{2} \in$ $\left(\xi_{2}(i), \xi_{3}(i)\right), \varepsilon \in\left(\varepsilon_{1}, \varepsilon_{2}\right), \tilde{\varepsilon}_{1} \in\left(\xi_{1}\left(i^{\prime}\right), \xi_{2}\left(i^{\prime}\right)\right), \tilde{\varepsilon}_{2} \in$ $\left(\xi_{2}\left(i^{\prime}\right), \xi_{3}\left(i^{\prime}\right)\right)$ and $\tilde{\varepsilon} \in\left(\tilde{\varepsilon}_{1}, \tilde{\varepsilon}_{2}\right)$, where $\xi_{1}(i), \xi_{2}(i)$, $\xi_{3}(i), \xi_{1}\left(i^{\prime}\right), \xi_{2}\left(i^{\prime}\right), \xi_{3}\left(i^{\prime}\right)$ are defined by Definition $1, i=2^{j}+k+1$ and $i^{\prime}=2^{j^{\prime}}+k^{\prime}+1$, it yields

$$
\left|\tilde{\varepsilon}_{4}-\tilde{\varepsilon}_{5}\right|,\left|\varepsilon_{1}-\varepsilon_{2}\right|,\left|\tilde{\varepsilon}_{1}-\tilde{\varepsilon}_{2}\right|<\frac{1}{2^{j^{\prime}}} .
$$

By using the same procedure, we can obtain the upper bound of $a_{1, i^{\prime}}$ when $i^{\prime}>1$ and that of $a_{i i^{\prime}}$ when $i, i^{\prime}>1$ as

$$
a_{1, i^{\prime}}=\frac{1}{2}\left(\tilde{\varepsilon}_{4}-\tilde{\varepsilon}_{5}\right) \frac{\partial}{\partial y} K\left(\varepsilon_{3}, \tilde{\varepsilon}_{6}\right)<\frac{\omega}{2^{j^{\prime}+1}},
$$

and

$$
\begin{aligned}
a_{i, i^{\prime}} & =\frac{1}{4}\left(\varepsilon_{1}-\varepsilon_{2}\right)\left(\tilde{\varepsilon}_{1}-\tilde{\varepsilon}_{2}\right) \frac{\partial^{2}}{\partial x \partial y} K(\varepsilon, \tilde{\varepsilon}) \\
& <\frac{\omega}{\left(2^{j+1}\right)\left(2^{j^{\prime}+1}\right)} .
\end{aligned}
$$

The next step is to determine the upper bound of the integrals in (50)-(52). By applying Theorem 1, we obtain

$$
\begin{aligned}
\mathscr{I}_{\Gamma}^{(1)} & \leqslant \frac{1}{(\Gamma !)^{2}}, \\
\mathscr{I}_{\Gamma ; j, k ; r, s}^{(2)} & <\frac{[C(\Gamma)]^{2}}{\left(2^{j+1}\right)^{2}\left(2^{r+1}\right)^{2}}, \\
\mathscr{I}_{\Gamma ; j, k}^{(3)} & <\frac{C(\Gamma)}{\Gamma !\left(2^{j+1}\right)^{2}},
\end{aligned}
$$

where $C(\Gamma)=8 /\left(3(\lfloor(\Gamma+1) / 2\rfloor)^{2}\right)$ and $\Gamma \geqslant 2$. Before determining the upper bound of $\mathscr{D}_{1}, \mathscr{D}_{2}, \mathscr{D}_{3}$, and $\mathscr{D}_{4}$, we require the following:

$$
\sum_{j=J+1}^{\infty} \sum_{k=0}^{2^{j}-1}\left(\frac{1}{2^{j+1}}\right)^{3}=\frac{1}{6}\left(\frac{1}{2^{J+1}}\right)^{2} .
$$

The next step is to find an upper bound of $\mathscr{D}_{1}$, $\mathscr{D}_{2}, \mathscr{D}_{3}$, and $\mathscr{D}_{4}$. By applying the upper bound of $a_{i i^{\prime}}$, (67)-(69) and (70) for (44)-(49), we obtain

$$
\begin{aligned}
\mathscr{D}_{1} & <\sum_{j, k} \sum_{r, s} \frac{[C(\Gamma)]^{2} \omega^{2}}{(\Lambda !)^{2}\left(2^{j+1}\right)^{3}\left(2^{r+1}\right)^{3}} \\
& \leqslant \frac{[C(\Gamma)]^{2} \omega^{2}}{36(\Lambda !)^{2}}\left(\frac{1}{2^{\tilde{J}+1}}\right)^{4}
\end{aligned}
$$

$$
\begin{aligned}
\mathscr{D}_{2} & <\sum_{j^{\prime}, k^{\prime}} \sum_{r^{\prime}, s^{\prime}} \frac{[C(\Lambda)]^{2} \omega^{2}}{(\Gamma !)^{2}\left(2^{j^{\prime}+1}\right)^{3}\left(2^{r^{\prime}+1}\right)^{3}} \\
& \leqslant \frac{[C(\Lambda)]^{2} \omega^{2}}{36(\Gamma !)^{2}}\left(\frac{1}{2^{\tilde{J}+1}}\right)^{4},
\end{aligned}
$$

$$
\begin{aligned}
& \mathscr{D}_{3}< \sum_{j, k} \sum_{r, s} \sum_{j^{\prime}, k^{\prime}} \sum_{r^{\prime}, s^{\prime}} \\
& \frac{[C(\Gamma)]^{2}[C(\Lambda)]^{2} \omega^{2}}{\left(2^{j+1}\right)^{3}\left(2^{r+1}\right)^{3}\left(2^{j^{\prime}+1}\right)^{3}\left(2^{r^{\prime}+1}\right)^{3}} \\
& \leqslant \frac{[C(\Gamma)]^{2}[C(\Lambda)]^{2} \omega^{2}}{1296}\left(\frac{1}{2^{\tilde{J}+1}}\right)^{8},
\end{aligned}
$$

$$
\begin{aligned}
\mathscr{D}_{4} & <2 \sum_{j, k} \sum_{r^{\prime}, s^{\prime}} \frac{C(\Gamma) C(\Lambda) \omega^{2}}{\Gamma ! \Lambda !\left(2^{j+1}\right)^{3}\left(2^{r^{\prime}+1}\right)^{3}} \\
& \leqslant \frac{C(\Gamma) C(\Lambda) \omega^{2}}{18 \Gamma ! \Lambda !}\left(\frac{1}{2^{\tilde{J}+1}}\right)^{4},
\end{aligned}
$$




$$
\begin{aligned}
\mathscr{D}_{5} & <2 \sum_{j, k} \sum_{r, s} \sum_{r^{\prime}, s^{\prime}} \frac{[C(\Gamma)]^{2} C(\Lambda) \omega^{2}}{\Lambda !\left(2^{j+1}\right)^{3}\left(2^{r+1}\right)^{3}\left(2^{r^{\prime}+1}\right)^{3}} \\
& \leqslant \frac{[C(\Gamma)]^{2} C(\Lambda) \omega^{2}}{108 \Lambda !}\left(\frac{1}{2^{\tilde{J}+1}}\right)^{6}, \\
\mathscr{D}_{6} & <2 \sum_{r, s} \sum_{j^{\prime}, k^{\prime}} \sum_{r^{\prime}, s^{\prime}} \frac{C(\Gamma)[C(\Lambda)]^{2} \omega^{2}}{\Gamma !\left(2^{r+1}\right)^{3}\left(2^{j^{\prime}+1}\right)^{3}\left(2^{r^{\prime}+1}\right)^{3}} \\
& \leqslant \frac{C(\Gamma)[C(\Lambda)]^{2} \omega^{2}}{108 \Gamma !}\left(\frac{1}{2^{\tilde{J}+1}}\right)^{6},
\end{aligned}
$$

where $j=J+1, \ldots, \infty ; k=0,1, \ldots, 2^{j}-1 ; r=J+$ $1, \ldots, \infty ; s=0,1, \ldots, 2^{r}-1 ; j^{\prime}=J^{\prime}+1, \ldots, \infty ; k^{\prime}=$ $0,1, \ldots, 2^{j^{\prime}}-1 ; r^{\prime}=J^{\prime}+1, \ldots, \infty ; s^{\prime}=0,1, \ldots, 2^{r^{\prime}}-$ 1 ; and $\tilde{J}=\min \left\{J, J^{\prime}\right\}$. By substituting (71)-(76) into (43), we have

$$
\begin{gathered}
{\left[\left\|E_{J J^{\prime}}(x, y)\right\|_{2}\right]^{2}} \\
<\frac{[C(\Gamma)]^{2} \omega^{2}}{36(\Lambda !)^{2}}\left(\frac{1}{2^{\tilde{J}+1}}\right)^{4}+\frac{[C(\Lambda)]^{2} \omega^{2}}{36(\Gamma !)^{2}}\left(\frac{1}{2^{\tilde{J}+1}}\right)^{4} \\
+\frac{[C(\Gamma)]^{2}[C(\Lambda)]^{2} \omega^{2}}{1296}\left(\frac{1}{2^{\tilde{J}+1}}\right)^{8} \\
+\frac{C(\Gamma) C(\Lambda) \omega^{2}}{18 \Gamma ! \Lambda !}\left(\frac{1}{2^{\tilde{J}+1}}\right)^{4} \\
+\frac{[C(\Gamma)]^{2} C(\Lambda) \omega^{2}}{108 \Lambda !}\left(\frac{1}{2^{\tilde{J}+1}}\right)^{6} \\
+\frac{C(\Gamma)[C(\Lambda)]^{2} \omega^{2}}{108 \Gamma !}\left(\frac{1}{2^{\tilde{J}+1}}\right)^{6} .
\end{gathered}
$$

Hence,

$$
\left\|E_{J J^{\prime}}(x, y)\right\|_{2}<G(\Gamma, \Lambda)\left(\frac{1}{2^{\tilde{J}+1}}\right)^{2},
$$

or

$$
\left\|E_{J J^{\prime}}(x, y)\right\|_{2}=\mathscr{O}\left(\left(\frac{1}{2^{\tilde{J}+1}}\right)^{2}\right) ; \Gamma, \Lambda \geqslant 2,
$$

where

$$
\begin{aligned}
G(\Gamma, \Lambda)= & \left\{\frac{[C(\Lambda)]^{2}}{36(\Gamma !)^{2}}+\frac{[C(\Gamma)]^{2}}{36(\Lambda !)^{2}}\right. \\
+ & \frac{[C(\Gamma)]^{2}[C(\Lambda)]^{2}}{1296}+\frac{C(\Gamma) C(\Lambda)}{18 \Gamma ! \Lambda !} \\
& \left.+\frac{[C(\Gamma)]^{2} C(\Lambda)}{108 \Lambda !}+\frac{C(\Gamma)[C(\Lambda)]^{2}}{108 \Gamma !}\right\}^{1 / 2} \omega,
\end{aligned}
$$

and $C(\Gamma)=8 /\left(3(\lfloor(\Gamma+1) / 2\rfloor !)^{2}\right)$ for $\Gamma \geqslant 2$.

\section{NUMERICAL VALIDATION}

In this section, the validation of the convergence analysis result is provided by the numerical results for Poisson and Helmholtz equations which are shown in examples 1 and 2, respectively. For convenience, we set the maximum level of resolution $J=J^{\prime}$, so $\tilde{J}=J=J^{\prime}$, and $M_{1}=M_{2}=2^{\tilde{J}}$. When estimating $E_{J J^{\prime}}(x, y)$, we consider the matrix equation (17) at the collocation points $x_{r}$ and $y_{s}$. Then, we introduce $E_{\tilde{J}}(x, y)$ as the error of approximation $E_{J J^{\prime}}(x, y)$ when $J=J^{\prime}=\tilde{J}$. By Theorem 2, we have the error of approximation at the maximal level of resolution $\tilde{J}$ as

$$
\left\|E_{\tilde{J}}(x, y)\right\|_{2} \sim\left(1 / 2^{\tilde{J}+1}\right)^{2} .
$$

Then we have

$$
\log _{2}\left(\left\|E_{\tilde{J}}(x, y)\right\|_{2} /\left\|E_{\tilde{J}+1}(x, y)\right\|_{2}\right) \sim 2 .
$$

Since the order of convergence from Theorem 2 is 2 , the order of convergence is

$$
\log _{2}\left(\left\|E_{\tilde{J}}(x, y)\right\|_{2} /\left\|E_{\tilde{J}+1}(x, y)\right\|_{2}\right) .
$$

The error of approximation from the numerical result can be obtained by

$$
\left(\left\|E_{\tilde{J}}(x, y)\right\|_{2}\right)_{\mathrm{num}}=\sqrt{\frac{\sum_{\Omega_{\mathrm{coll}}}\left(E_{\tilde{J}}\left(x_{r}, y_{s}\right)\right)^{2}}{n\left(\Omega_{\mathrm{coll}}\right)}},
$$

where $\Omega_{\text {coll }}$ is the set of collocation points $\left(x_{r}, y_{s}\right)$ and $n\left(\Omega_{\text {coll }}\right)$ is the number of collocation points.

\section{Poisson equation}

For convenience, we introduce the Poisson equation with the same boundary conditions as in Ref. 7 (see equation (26) in Ref. 7) by considering the Poisson equation as

$$
\frac{\partial^{2}}{\partial x^{2}} u(x, y)+\frac{\partial^{2}}{\partial y^{2}} u(x, y)=f(x, y)
$$

where $(x, y) \in[0,1]^{2}$, with the boundary conditions $u(x, 0)=u(0, y)=u(x, 1)$ and $u(1, y)=g(y)$. Then let

$$
\frac{\partial^{4}}{\partial x^{2} \partial y^{2}} u(x, y)=\sum_{i=1}^{2 M_{1}} \sum_{i^{\prime}=1}^{2 M_{2}} a_{i i^{\prime}} h_{i}(x) h_{i^{\prime}}(y) .
$$

According to Lepik ${ }^{7}$, by integrating twice each with respect to $x$ and $y$ and incorporating the boundary 
conditions, we obtain the numerical solution via

$$
\begin{array}{r}
u(x, y)=\sum_{i=1}^{2 M_{1}} \sum_{i^{\prime}=1}^{2 M_{2}} a_{i i^{\prime}}\left[p_{2, i}(x) p_{2, i^{\prime}}(y)\right. \\
-y p_{2, i}(x) p_{2, i^{\prime}}(1)-x p_{2, i}(1) p_{2, i^{\prime}}(y) \\
\left.+x y p_{2, i}(1) p_{2, i^{\prime}}(1)\right]+x g(y) .
\end{array}
$$

Then,

$$
\begin{aligned}
\frac{\partial^{2}}{\partial x^{2}} u(x, y)= & \sum_{i=1}^{\infty} \sum_{i^{\prime}=1}^{\infty} a_{i i^{\prime}}\left[h_{i}(x) p_{2, i^{\prime}}(y)\right. \\
& \left.-h_{i}(x) y p_{2, i^{\prime}}(1)\right], \\
\frac{\partial^{2}}{\partial y^{2}} u(x, y)= & \sum_{i=1}^{\infty} \sum_{i^{\prime}=1}^{\infty} a_{i i^{\prime}}\left[p_{2, i}(x) h_{i^{\prime}}(y)\right. \\
& \left.-x p_{2, i}(1) h_{i^{\prime}}(y)\right]+x g^{\prime \prime}(y) .
\end{aligned}
$$

By substituting (81) and (83) into (78), we have the matrix equation in the form of (17) as

$$
\sum_{i=1}^{2 M_{1}} \sum_{i^{\prime}=1}^{2 M_{2}} a_{i i^{\prime}} R_{i i^{\prime} l l^{\prime}}=f_{l l^{\prime}},
$$

where

$$
\begin{aligned}
R_{i i^{\prime} l l^{\prime}}=h_{i}( & \left.x_{l}\right) p_{2, i^{\prime}}\left(y_{l^{\prime}}\right)+p_{2, i}\left(x_{l}\right) h_{i^{\prime}}\left(y_{l^{\prime}}\right) \\
& -h_{i}\left(x_{l}\right) y_{l^{\prime}} p_{2, i^{\prime}}(1)-x_{l} p_{2, i}(1) h_{i^{\prime}}\left(y_{l^{\prime}}\right)
\end{aligned}
$$

and $f_{l l^{\prime}}=f\left(x_{l}, y_{l^{\prime}}\right)-x_{l} g^{\prime \prime}\left(y_{l^{\prime}}\right)$. The numerical solution can be obtained by calculating $a_{i i^{\prime}}$ and substituting back into (79).

Example 1 The Poisson equation

$$
\begin{aligned}
\nabla^{2} u(x, y)=2\left[y^{2}\left(1-6 x^{2}\right)\left(1-y^{2}\right)\right. \\
\left.+x^{2}\left(1-6 y^{2}\right)\left(1-x^{2}\right)\right],
\end{aligned}
$$

with the boundary conditions that $u(x, 0)=$ $u(0, y)=u(x, 1)=u(1, y)=0$, has the exact solution $u_{\mathrm{ex}}(x, y)$ in the form

$$
u_{\mathrm{ex}}(x, y)=x^{2} y^{2}\left(1-x^{2}\right)\left(1-y^{2}\right) .
$$

Numerical errors from solving the Poisson equation using the Haar wavelet method are shown in Table 1.

\section{Helmholtz equation}

To exploit the calculation of the Poisson equation for obtaining the general solution in the form (15), we introduce the Helmholtz equation

$$
\nabla^{2} u(x, y)+k^{2} u(x, y)=f(x, y),
$$

Table 1 Numerical results for Example 1.

\begin{tabular}{lrr}
\hline$\tilde{J}$ & numerical error & order \\
\hline 1 & $6.19 \times 10^{-4}$ & \\
2 & $1.67 \times 10^{-4}$ & 1.8940 \\
3 & $4.24 \times 10^{-5}$ & 1.9731 \\
4 & $1.07 \times 10^{-5}$ & 1.9932 \\
5 & $2.67 \times 10^{-6}$ & 1.9983 \\
\hline
\end{tabular}

where $(x, y) \in[0,1]^{2}$, with the boundary conditions $u(x, 0)=u(0, y)=u(x, 1)$ and $u(1, y)=g(y)$. Then let

$$
\frac{\partial^{4}}{\partial x^{2} \partial y^{2}} u(x, y)=\sum_{i=1}^{2 M_{1}} \sum_{i^{\prime}=1}^{2 M_{2}} a_{i i^{\prime}} h_{i}(x) h_{i^{\prime}}(y) .
$$

Since (85) has the same boundary condition as the Poisson equation in (78), by the same procedure, we have the general solution of the Helmholtz equation $u(x, y)$, and derivatives $u_{x x}(x, y)$ and $u_{y y}(x, y)$ the same as (79), (81) and (83), respectively. By substituting (79), (81) and (83) into (85), we have the matrix equation in the form of (17) as

$$
\sum_{i=1}^{2 M_{1}} \sum_{i^{\prime}=1}^{2 M_{2}} a_{i i^{\prime}} R_{i i^{\prime} l l^{\prime}}=f_{l l^{\prime}}
$$

where

$$
\begin{aligned}
R_{i i^{\prime} l l^{\prime}}= & h_{i}\left(x_{l}\right) p_{2, i^{\prime}}\left(y_{l^{\prime}}\right)+p_{2, i}\left(x_{l}\right) h_{i^{\prime}}\left(y_{l^{\prime}}\right) \\
& \quad-h_{i}\left(x_{l}\right) y_{l^{\prime}} p_{2, i^{\prime}}(1)-x_{l} p_{2, i}(1) h_{i^{\prime}}\left(y_{l^{\prime}}\right) \\
+ & k^{2}\left[p_{2, i}\left(x_{l}\right) p_{2, i^{\prime}}\left(y_{l^{\prime}}\right)-y_{l^{\prime}} p_{2, i}\left(x_{l}\right) p_{2, i^{\prime}}(1)\right. \\
& \left.\quad-x_{l} p_{2, i}(1) p_{2, i^{\prime}}\left(y_{l^{\prime}}\right)+x_{l} y_{l^{\prime}} p_{2, i}(1) p_{2, i^{\prime}}(1)\right],
\end{aligned}
$$

and $f_{l l^{\prime}}=f\left(x_{l}, y_{l^{\prime}}\right)-x_{l} g^{(2)}\left(y_{l^{\prime}}\right)-x_{l} k^{2} g\left(y_{l^{\prime}}\right)$. The numerical solution can be obtained by the same procedure as in the case of the Poisson equation.

Example 2 The Helmholtz equation

$$
\begin{aligned}
& \nabla^{2} u(x, y)+k^{2} u(x, y) \\
& =\left(k^{2}-2 \pi^{2}\right) \sin (\pi x) \sin (\pi y),
\end{aligned}
$$

with the boundary conditions $u(x, 0)=u(0, y)=$ $u(x, 1)=u(1, y)=0$ has the exact solution

$$
u_{\mathrm{ex}}(x, y)=\sin (\pi x) \sin (\pi y) .
$$

Numerical errors from solving the Helmholtz equation using the Haar wavelet method (when $k=0.5$ ) are shown in Table 2. 
Table 2 Numerical results for Example $2(k=0.5)$.

\begin{tabular}{lrr}
\hline$\tilde{J}$ & numerical error & order \\
\hline 1 & $1.04 \times 10^{-2}$ & \\
2 & $2.63 \times 10^{-3}$ & 1.9840 \\
3 & $6.59 \times 10^{-4}$ & 1.9969 \\
4 & $1.65 \times 10^{-4}$ & 1.9993 \\
5 & $4.12 \times 10^{-5}$ & 1.9998 \\
\hline
\end{tabular}

\section{CONCLUSIONS}

Theorem 2 shows that the method ${ }^{7}$ based on the two-dimensional Haar wavelet converges as the maximum level of resolution increases. The convergence analysis shows that the order of approximation is 2 for boundary value problems.

Acknowledgements: This study is supported by the Faculty of Science, Mahidol University for the Sri-Trangthong scholarship. We thank Michael A. Allen for his invaluable assistance in language advice.

\section{REFERENCES}

1. Chen CF, Hsiao CH (1997) Haar wavelet method for solving lumped and distributed-parameter systems. IEE Proc Contr Theor Appl 144, 87-94.

2. Hsiao CH (1997) State analysis of linear time delayed systems via Haar wavelets. Math Comput Simul 44, $457-70$.

3. Lepik Ü (2005) Numerical solution of differential equations using Haar wavelets. Math Comput Simul $68,127-43$.

4. Lepik Ü (2007) Numerical solution of evolution equations by the Haar wavelet method. Appl Math Comput 185, 695-704.

5. Lepik Ü (2008) Solving integral and differential equations by the aid of non-uniform Haar wavelets. Appl Math Comput 198, 326-32.

6. Lepik Ü (2008) Haar wavelet method for solving higher order differential equations. Int $J$ Math Comput 1, 84-94.

7. Lepik Ü (2011) Solving PDEs with the aid of twodimensional Haar wavelets. Comput Math Appl 61, 1873-9.

8. Siraj-ul-Islam, Šarler B, Aziz I, Fazal-i-Haq (2011) Haar wavelet collocation method for the numerical solution of boundary layer fluid flow problems. Int $J$ Therm Sci 50, 686-97.

9. Siraj-ul-Islam Aziz I, Ahmad M (2015) Numerical solution of two-dimensional elliptic PDEs with nonlocal boundary conditions. Comput Math Appl 69, 180-205.

10. Majak J, Shvartsman BS, Kirs M, Pohlak M, Herranen H (2015) Convergence theorem for the Haar wavelet based discretization method. Compos Struct 126, $227-32$.
11. Majak J, Shvartsman B, Karjust K, Mikola M, Haavajõe A, Pohlak M (2015) On the accuracy of the Haar wavelet discretization method. Composites $B$ 80, 321-7.

12. Podlubny I (1998) Fractional Differential Equations: An Introduction to Fractional Derivatives, Fractional Differential Equations, to Methods of their Solution and some of their Applications, Mathematics in Science and Engineering vol 198, Academic Press, San Diego, CA. 